\title{
Narrowing Constraints with Type Ia Supernovae: Converging on a Cosmological Constant
}

\author{
Scott Sullivan ${ }^{1}$, Asantha Cooray ${ }^{1}$, Daniel E. Holz ${ }^{2,3}$ \\ ${ }^{1}$ Center for Cosmology, Department of Physics \& Astronomy, University of \\ California, Irvine, 92697 \\ ${ }^{2}$ Theoretical Division, Los Alamos National Laboratory, Los Alamos, NM 87545 \\ ${ }^{3}$ Department of Astronomy \& Astrophysics, University of Chicago, Chicago, IL \\ 60637
}

\begin{abstract}
We apply a parameterization-independent approach to fitting the dark energy equation-of-state (EOS). Utilizing the latest type Ia supernova data, combined with results from the cosmic microwave background and baryon acoustic oscillations, we find that the dark energy is consistent with a cosmological constant. We establish independent estimates of the evolution of the dark energy EOS by diagonalizing the covariance matrix. We find three independent constraints, which taken together imply that the equation of state is more negative than -0.2 at the $68 \%$ confidence level in the redshift range $0<z<1.8$, independent of the flat universe assumption. Our estimates argue against previous claims of dark energy "metamorphosis," where the EOS was found to be strongly varying at low redshifts. Our results are inconsistent with extreme models of dynamical dark energy, both in the form of "freezing" models where the dark energy EOS begins with a value greater than -0.2 at $z>1.2$ and rolls to a value of -1 today, and "thawing" models where the EOS is near -1 at high redshifts, but rapidly evolves to values greater than -0.85 at $z<0.2$. Finally, we propose a parameterization-independent figure-of-merit, to help assess the ability of future surveys to constrain dark energy. While previous figures-of-merit presume specific dark energy parameterizations, we suggest a binning approach to evaluate dark energy constraints with a minimum number of assumptions.
\end{abstract}




\section{Introduction}

Distance estimates to Type Ia supernovae (SNe) are currently a preferred probe of the expansion history of the Universe [1], and have led to the discovery that the expansion is accelerating [2, 3, 4, 5]. It is now believed that a mysterious dark energy component, with an energy density $\sim 70 \%$ of the total energy density of the universe, is responsible for the accelerated expansion [6, 7]. While the presence of acceleration is now well established by various cosmological probes, the underlying physics remains a complete mystery [8]. As the precise nature of the dark energy has profound implications, understanding its properties is one of the biggest challenges of modern physics.

With the advent of large surveys for Type Ia supernovae, such as the Supernova Legacy Survey (SNLS)† [9] and Essence\&] [4, among others, it is now possible to consider detailed studies of the expansion history of the Universe, and shed light on the underlying physics responsible for the acceleration. Although the dark energy may be complex, thus far it is generally described by a cosmological constant, or through a simple dynamical component such as a single scalar field rolling down a potential [10]. The observational data is then used to constrain these simple models, generally in the from of determining a dark energy equation-of-state (EOS) describing the ratio of its pressure to its density [11], or by measuring dynamical parameters such as the cosmic jerk [12]. Using the EOS as the primary variable, several studies have considered how current and future data might be used to make statements on the physics responsible for dark energy [13], including attempts to establish the shape of the scalar field potential [14, 15].

When model fitting data it is generally assumed that the dark energy EOS as a function of redshift, $w(z)$, follows a certain predetermined evolutionary history. Common parameterizations include a linear variations with redshift, $w(z)=w_{0}+w_{z} z$ [16], an evolution that asymptotes to a constant $w$ at high redshift, $w(a)=w_{0}+w_{a}(1-a)$ with $a$ as the scale factor [17], or an evolution with an EOS of the form $w(z)=$ $w_{0}-\alpha \ln (1+z)$ [18]. Unfortunately, fitting data to an assumed functional form leads to possible biases in statements one makes about the dark energy and its evolution, especially if the true behavior of the dark energy EOS differs significantly from the assumed functional form [15]. Moreover, statements related to the dark energy EOS are often made under the assumption of a spatially flat universe, while there still exists percent-level uncertainties on the curvature.

Instead of using a parameterized form for $w(z)$, one can also utilize a variant of the principal component analysis advocated in Ref. [19] to establish the EOS with redshift. This was first applied in Ref. [20] to a set of supernova data from Ref. [1]. Recently, Riess et al. 3] analyzed a new set of $z>1$ SNe from the Hubble Space Telescope combined with low redshift SNe, and by making use of the same technique of decorrelating the $w_{i}$ binned estimates, established that the EOS is not strongly evolving. Since the analysis of Riess et al. [3], the supernovae sample size has increased by at least by a factor of 2

$\ddagger$ http://www.cfht.hawaii.edu/SNLS/

$\S$ http://www.ctio.noao.edu/w̃sne/ 
through the Essence survey [4]. The SNe light curves from several independent datasets have been analyzed with a common method to extract distance moduli in Ref. [5], and we use this publicly available data $\llbracket$ to extract the EOS.

We combine the different distance measurements to extract independent estimates of the EOS when binned in several redshift bins in the redshift range $0<z<1.8$. We make use of these binned and uncorrelated estimates of the EOS to address a simple question: is the dark energy consistent with a cosmological constant? For a cosmological constant $w(z)=-1$ exactly, while dynamical dark energy models lead to an EOS that either evolves from a large value to -1 today (models which are categorized as "freezing" in Ref. [21]) or from -1 at high redshift to large values today ("thawing" models of Ref. [21]). In our analysis we also allow departures from a flat universe, but allow curvature to be constrained based on complementary information from the cosmic microwave background (WMAP; [7]) and baryon acoustic oscillation distance scales [26]. As discussed in Ref. [20], our analysis is facilitated by the fact that our measurements of the EOS are completely uncorrelated. Although we focus on the EOS, we note that one can also extract uncorrelated estimates of other parameters related to the expansion history and dark energy, such as the dark energy density [22]. However, unlike the case with the EOS, such estimates cannot be used to directly address the simple question of whether the dark energy is a cosmological constant.

We make use of our binned estimates to comment on several new developments related to dark energy studies. First, in addition to addressing the extent to which $w(z)=-1$ in present data, we also comment on the extent to which dynamical dark energy models, such as "freezing" and "thawing" models [21], may be distinguished or ruled out with current cdistance data. We also discuss the role uncorrelated, independent EOS measurements can play in furthering our understanding of the dark energy. Recently, the Dark Energy Task Force (DETF) [23] has suggested a figure-ofmerit to compare the abilities of different experiments to extract information related to dark energy. This is done in terms of the inverse area of the error ellipse of the equation of state and its evolution with redshift, utilizing either the Linder parameterization [17] or with two parameters of the form $w(a)=w_{p}+\left(a_{p}-a\right) w_{a}$ [13]. The discussion is then restricted to a two parameter description of the dark energy equation of state, assuming a very specific evolutionary behavior. The ability to extract information about dark energy from current and future experiments thus becomes a model dependent statement. Using uncorrelated, independent equation of state estimates, we propose a model independent figure-of-merit. Our approach involves the inverse of the sum of inverse variances of uncorrelated $w\left(z_{i}\right)$ bins as an way to capture all of the available information related to dark energy in a given data set or experiment.

The paper is organized as follows: In the next Section we review techniques for reconstructing the EOS, we following the methods of Ref. [20] and Ref. [3]. In Section 3 we present our results, addressing whether dark energy is a cosmological constant or has || http://www.ctio.noao.edu/wproject/wresults/ 


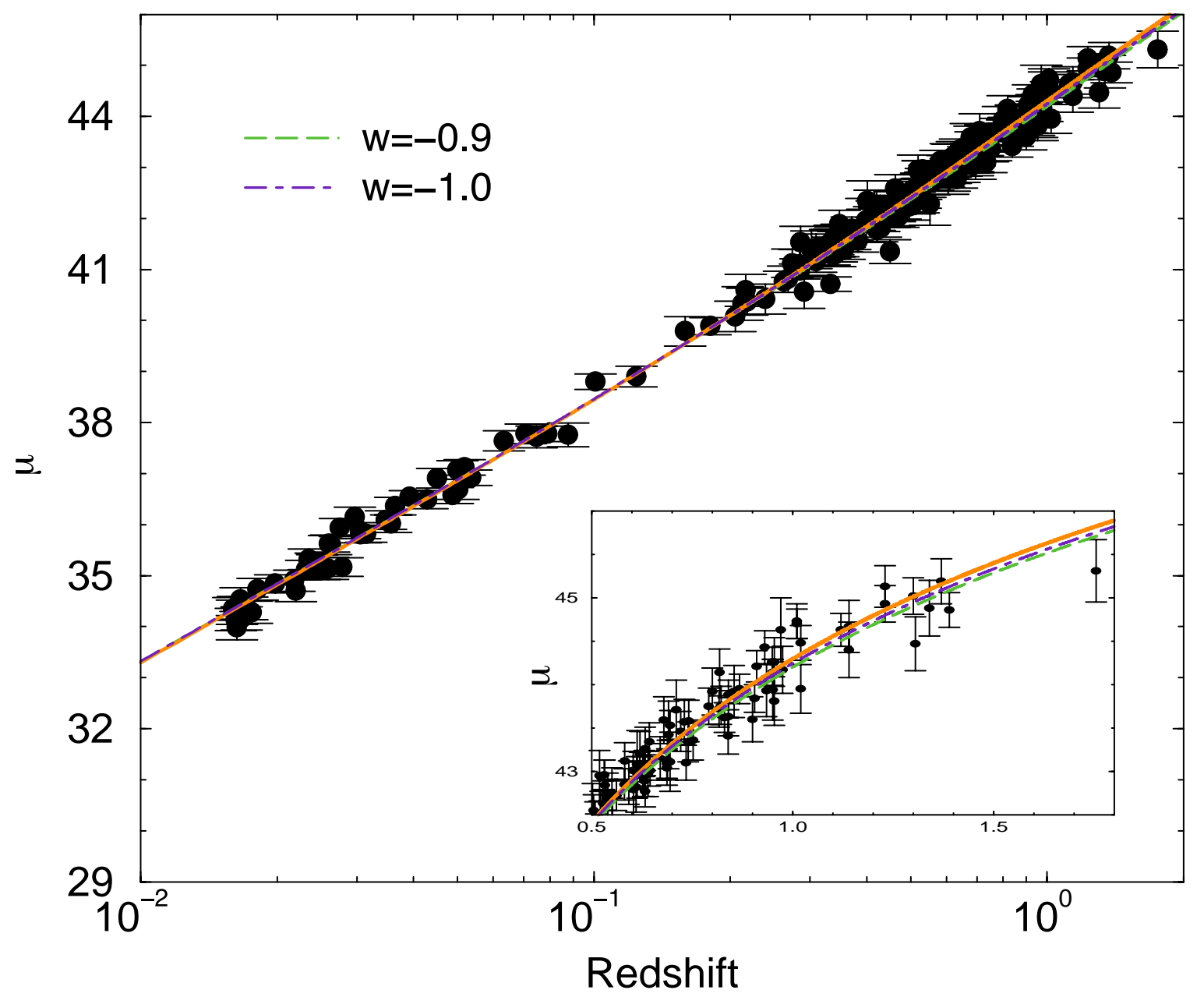

Figure 1. Hubble diagram for type Ia supernova data used for the present analysis. The dataset includes a total of 192 SNe from the recent analysis of light curves in Ref. [5]. For comparison, we also separately analyze a subset of $104 \mathrm{SNe}$ within this sample that were also used in Riess et al. 3]. The distance moduli used here for this subset, however, are different from this original study due to the reanalysis of light curves in Davis et al. [5. The orange line is for our best fit.

dynamical behavior that leads to an evolution in the EOS. We show that existing data rule out extreme forms of dynamical behavior at the $68 \%$ confidence level, including models that either start with $w(z)>-0.4$ at $z>1.0$ or models that asymptote to values of $w(z)>-0.85$ at $z<0.2$. In Section 3.1 we outline a new figure of merit to assess the dark energy information content of future experiments, based on uncorrelated binned estimates of the EOS. We conclude with a summary of results in Section 4.

\section{Methodology}

A simple way to model the dark component of the universe credited for the accelerating expansion is through a modification of the standard cosmological model. We utilize the Friedmann equations, and specify the dark energy density and its equation-of-state 
(EOS). We assume a piecewise constant EOS, with value $w_{i}$ in each $i^{\text {th }}$ redshift bin (defined by an upper boundary at $z_{i}$ ). We fit the observational data to the luminosity distance as a function of redshift. The expression for luminosity distance, $d_{L}(z)$, depends on whether the universe is flat, positively, or negatively curved (i.e., the sign of $\Omega_{k}$ ), and is given by

$$
d_{L}(z)=(1+z) \frac{c}{H_{0}} \times \begin{cases}\frac{1}{\sqrt{\left|\Omega_{k}\right|}} \sinh \left(\sqrt{\left|\Omega_{k}\right|} \int_{0}^{z} \frac{d z^{\prime}}{E\left(z^{\prime}\right)}\right) & \text { if } \Omega_{k}>0 \\ \int_{0}^{z} \frac{d z^{\prime}}{E\left(z^{\prime}\right)} & \text { if } \Omega_{k}=0 \\ \frac{1}{\sqrt{\left|\Omega_{k}\right|}} \sin \left(\sqrt{\left|\Omega_{k}\right|} \int_{0}^{z} \frac{d z^{\prime}}{E\left(z^{\prime}\right)}\right) & \text { if } \Omega_{k}<0\end{cases}
$$

where

$$
E(z)=\left[\Omega_{M}(1+z)^{3}+\Omega_{k}(1+z)^{2}+\left(1-\Omega_{k}-\Omega_{M}\right) F(z)\right]^{\frac{1}{2}},
$$

and $F(z)$ depends on the binning of $w(z)$. For the $n^{\text {th }}$ redshift bin $F(z)$ has the form

$$
F\left(z_{n}>z>z_{n-1}\right)=(1+z)^{3\left(1+w_{n}\right)} \prod_{i=0}^{n-1}\left(1+z_{i}\right)^{3\left(w_{i}-w_{i+1}\right)} .
$$

We define the zeroth bin as $z_{0}=0$, so the product is unity for redshift $z$ in the first bin. For our primary analysis we set $z_{1}=0.2, z_{2}=0.5, z_{3}=1.8$, and $z_{4}$ extends beyond the surface of last scattering at $z_{C M B}=1089$. We assume $w(z>1.8)=-1$ and allow variation within the remaining three redshift bins. Selecting the cutoff point for $z_{3}$ is fairly arbitrary; we found that pushing it back as far as $z_{3}=2.5$ does not substantially alter the outcome of our analysis.

In addition to SNe, we also make use of four primary constraints from the literature following the analysis of Riess et al. [3], modifying these to account for variations in curvature since the analysis in Ref. [3] assumed an a priori flat cosmological model. These constraints are:

- The product of the Hubble parameter $h \equiv H_{0} / 100$ and the present local mass density $\Omega_{m}$ from SDSS large scale structure measurements [38, given by $\Omega_{m} h=$ $0.213 \pm 0.023$. In cases where we allow curvature to vary, we either take a flat, broad prior in curvature or, to highlight the result under the assumption of a measured value for the curvature, we take $\Omega_{k}=-0.014 \pm 0.017$ as derived by WMAP analysis by combining WMAP and the Hubble constant [7].

- The SDSS luminous red galaxy baryon acoustic oscillation (BAO) distance estimate to redshift $z_{\mathrm{BAO}}=0.35$. Here the constraint is on the overall parameter $A \equiv$ $\frac{\sqrt{\Omega_{M} H_{0}^{2}}}{c z_{\mathrm{BAO}}}\left[r^{2}\left(z_{\mathrm{BAO}}\right) \frac{c z_{\mathrm{BAO}}}{H_{0} E\left(z_{\mathrm{BAO}}\right)}\right]^{1 / 3}$, where $r(z)=d_{L}(z) /(1+z)$ is the angular diameter distance. The angular correlation function of red galaxies in the SDSS spectroscopy survey leads to $A=0.469\left(\frac{n}{0.98}\right)^{-0.35} \pm 0.017$ [26]. Following Riess et al. [3], we use the WMAP estimate for the scalar tilt with $n=0.95$ [7].

- The distance to last scattering, at $z_{\mathrm{CMB}}=1089$, written in the dimensionless form $R_{C M B} \equiv \frac{\sqrt{\Omega_{M} H_{0}^{2}}}{c} r\left(z_{\mathrm{CMB}}\right)$ where $r\left(z_{\mathrm{CMB}}\right)$ is the angular diameter distance to the CMB last scattering surface. We use the dark energy and curvature independent estimate with $R=1.70 \pm 0.03$ [27]. 
- The distance ratio between $z=0.35$ and last scattering $z=1089$ as measured by the SDSS BAO analysis [26]:

$$
R_{0.35}=\frac{\left[r^{2}\left(z_{\mathrm{BAO}}\right) \frac{c z_{\mathrm{BAO}}}{H_{0} E\left(z_{\mathrm{BAO}}\right)}\right]^{1 / 3}}{r\left(z_{\mathrm{CMB}}\right)},
$$

with the value of $R_{0.35}=0.0979 \pm 0.0036$.

When estimating parameters we make use of the $\chi^{2}$ statistic for a particular model with parameter set $\theta\left(w_{i}, H_{0}, \Omega_{m}\right.$, and in some cases $\left.\Omega_{k}\right)$ :

$$
\chi^{2}(\theta)=\sum_{n=1}^{N}\left(\frac{\mu_{i}^{\text {theory }}-\mu_{i}^{\text {data }}}{\sigma_{i}^{2}+\sigma_{\text {int }}^{2}}\right),
$$

where $N$ is the total number of supernovae in the sample. While our total sample includes 192 supernovae, we also extract a subset of 104 supernovae that was analyzed previously [3]. While the subsample is for comparison with previous results, our distance estimates differ from the original analysis due to a reanalysis of light curves by Davis et al. [5] using a common light curve fitting method. When estimating $\chi^{2}$ we set an intrinsic dispersion of order $\sigma_{\text {int }} \sim 0.1$, such that the minimum $\chi^{2}$ value for the best fit model comes to be about one. Using $\chi^{2}$ we calculate the probability $P(\theta) \propto \exp \left(-\frac{\chi^{2}(\theta)}{2}\right)$ and derive constraints by generating Markov chains through a Monte-Carlo algorithm. The algorithm generates a set of models whose members appear in the set (or chain) a number of times proportional to their likelihood of being a good fit to the observed data, after marginalizing over other priors. The likelihood probability functions for each independent parameter are generated by simply taking a histogram over the chain. We marginalize over $H_{0}$ assuming a broad uniform prior over the range $[30,85] \mathrm{km} \mathrm{s}^{-1}$ $\mathrm{Mpc}^{-1}$. We also marginalize over $\Omega_{m}$ assuming the quoted prior above for $\Omega_{m} h$ using SDSS large scale structure measurements.

In the case of dark energy parameters, the redshift binned EOS estimates are correlated such that their errors depend upon each other. These correlations in the redshift binned EOS estimates, $w_{i}$, are captured by the covariance matrix, and this matrix can be generated by taking the average over the chain such that:

$$
C=\left\langle\mathbf{w} \mathbf{w}^{T}\right\rangle-\langle\mathbf{w}\rangle\left\langle\mathbf{w}^{T}\right\rangle .
$$

This covariance matrix is not diagonal as the values found for the various EOS estimates are correlated. This is expected, as the integration over low redshift bins in Eq. (1) obviously affects the model fit in middle and higher redshift bins. The behavior is also such that the best constraints are found for the lowest redshift bin, with higher bins having progressively weaker constraints. With the addition of the distance scale to the last scattering surface from CMB data, the constraint in the higher redshift bins are improved, as seen in prior analyses [3].

Instead of discussing $w(z)$ in correlated bins, we follow Huterer \& Cooray [20] and transform the covariance matrix to decorrelate the EOS estimates. This is achieved 
by changing the basis through an orthogonal matrix rotation that diagonalizes the covariance matrix. We start by the definition of the Fisher matrix,

$$
\mathbf{F} \equiv C^{-1}=\mathrm{O}^{\mathrm{T}} \Lambda \mathrm{O}
$$

where the matrix $\Lambda$ is the diagonalized covariance for transformed bins. The uncorrelated parameters are then defined by the rotation performed by the orthogonal rotation matrix $\mathbf{q}=\mathbf{O} \mathbf{w}$ and has the covariance matrix of $\Lambda^{-1}$. There is freedom of choice in what orthogonal matrix is used to perform this transformation, and we use the particular choice that was advocated in Ref. [20] and write the weight transformation matrix:

$$
\tilde{W}=\mathbf{O}^{T} \Lambda^{\frac{1}{2}} \mathbf{O}
$$

where the rows are then summed such that the weights from each band add up to unity. This choice ensures we have mostly positive contributions across all bands, an intuitively pleasing result, so for example we can interpret the weighting matrix as an indication of how much a measurement in the third bin is influenced by SNe in the first and second bins. We apply the transformation $\tilde{W}$ to each link in the Markov chain to generate a set of independent, uncorrelated measures of the EOS and its probability distributions as determined by the observables.

In addition to probability distribution functions for each of the uncorrelated EOS estimates, to study the redshift evolution of $w(z)$, we also study the differences between these uncorrelated estimates. The probability distribution functions of the difference between estimate $w_{i}$ and $w_{j}$ is generated through $P\left(w_{\text {diff }}\right) \propto \int P_{w_{i}}(w) P_{w_{j}}\left(w+w_{\text {diff }}\right) d w$. While in the uncorrelated case an estimate $w_{i}$ is not necessarily associated with a single redshift bin, due to support from adjacent redshift bins due to the transformation, any significant difference between $w_{i}$ and $w_{j}$ can be considered to be evidence for an evolution in the dark energy EOS. Moreover, if the dark energy is associated with a cosmological constant, then these difference estimates should be precisely zero.

\section{Results}

The results presented in this paper are derived from the statistical analysis of a combination of recent supernova surveys, including the Supernova Legacy Survey (SNLS) [9], the ESSENCE survey [4], and high- $z$ supernovae discovered by the Hubble Space Telescope (HST) 3]. In particular, we use a total of $192 \mathrm{SNe}$ Ia measurements taken from a combination of supernovae analyzed in Ref. [5] using a common light curve fitting method (Figure 1). Also, for comparison, results are presented for the $104 \mathrm{SNe}$ Ia that overlap with the "Gold" data set presented in Riess et al. [3], although the distance moduli values we use here for the same subsample are slightly different from the values published in the original analysis due to variations in the light curve fitting. This analysis includes the four external constraints outlined in the previous section. In addition to the standard flat cosmological model generally assumed when making fits 

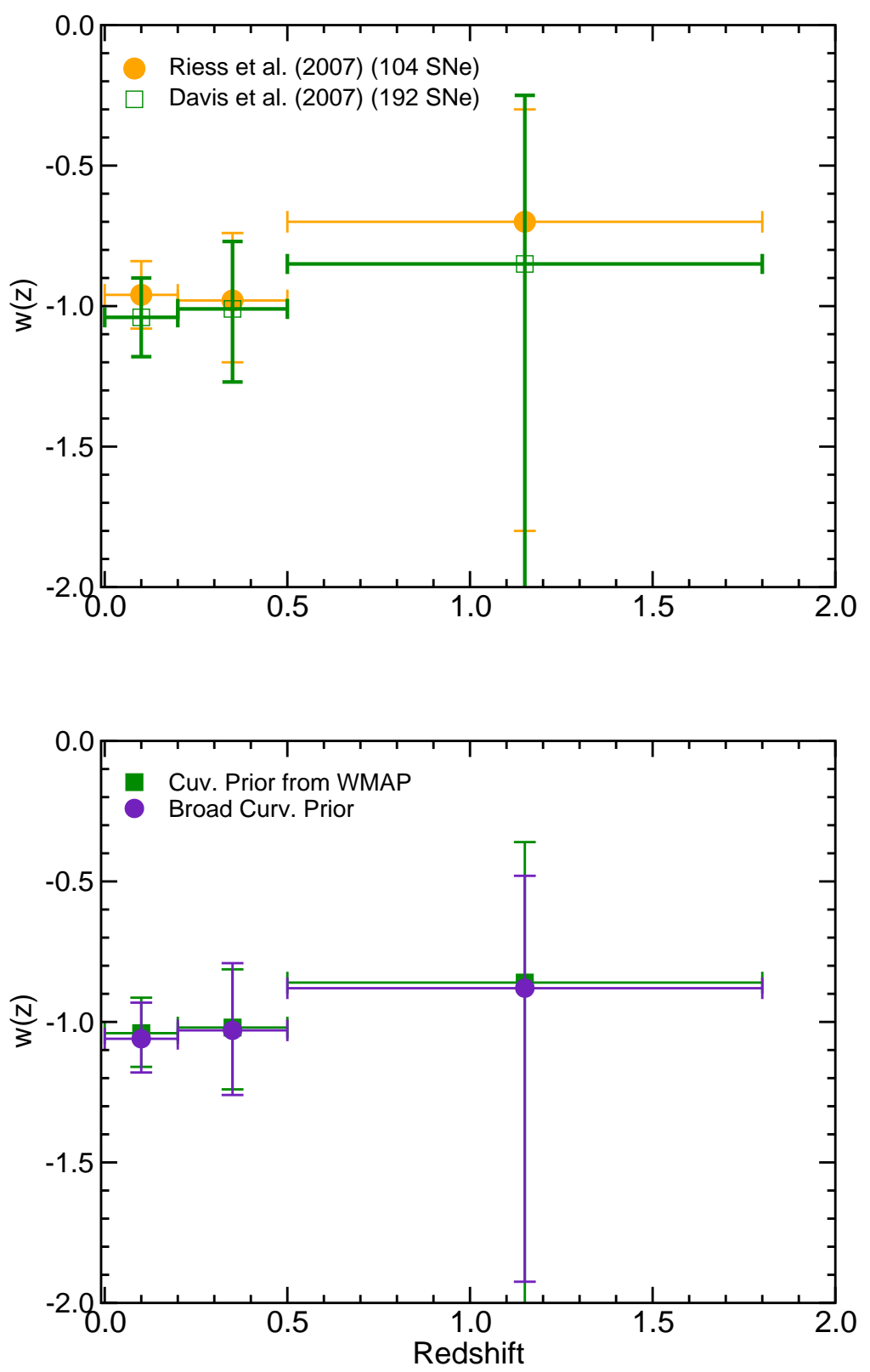

Figure 2. Uncorrelated estimates of the dark energy equation of state using a combined sample of supernova data and constraints from WMAP and BAO measurements. In the top panel a flat universe prior is assumed $\left(\Omega_{k}=0\right)$, and the filled and open symbols show the constraint with the total sample of $192 \mathrm{SNe}$ from Ref. [5] and a subset of $104 \mathrm{SNe}$ corresponding to a previous analysis [3], respectively. In the bottom panel we show $w(z)$ estimates without the flat universe prior; open and filled symbols showing constraints with a broad prior for $\Omega_{k}$ and $\Omega_{k}=-0.014 \pm 0.017$, respectively.

to dark energy EOS, we also allow for variations in the curvature, both with a prior on $\Omega_{k}$ based on WMAP and Hubble constant measurements, and with no prior.

In Figure 2 we highlight our results for $w(z)$, in the redshift bins $z<0.2$, $0.2<z<0.5$ and $1.8>z>0.5$, with $w(z>1.8)=-1$. These binned estimates 

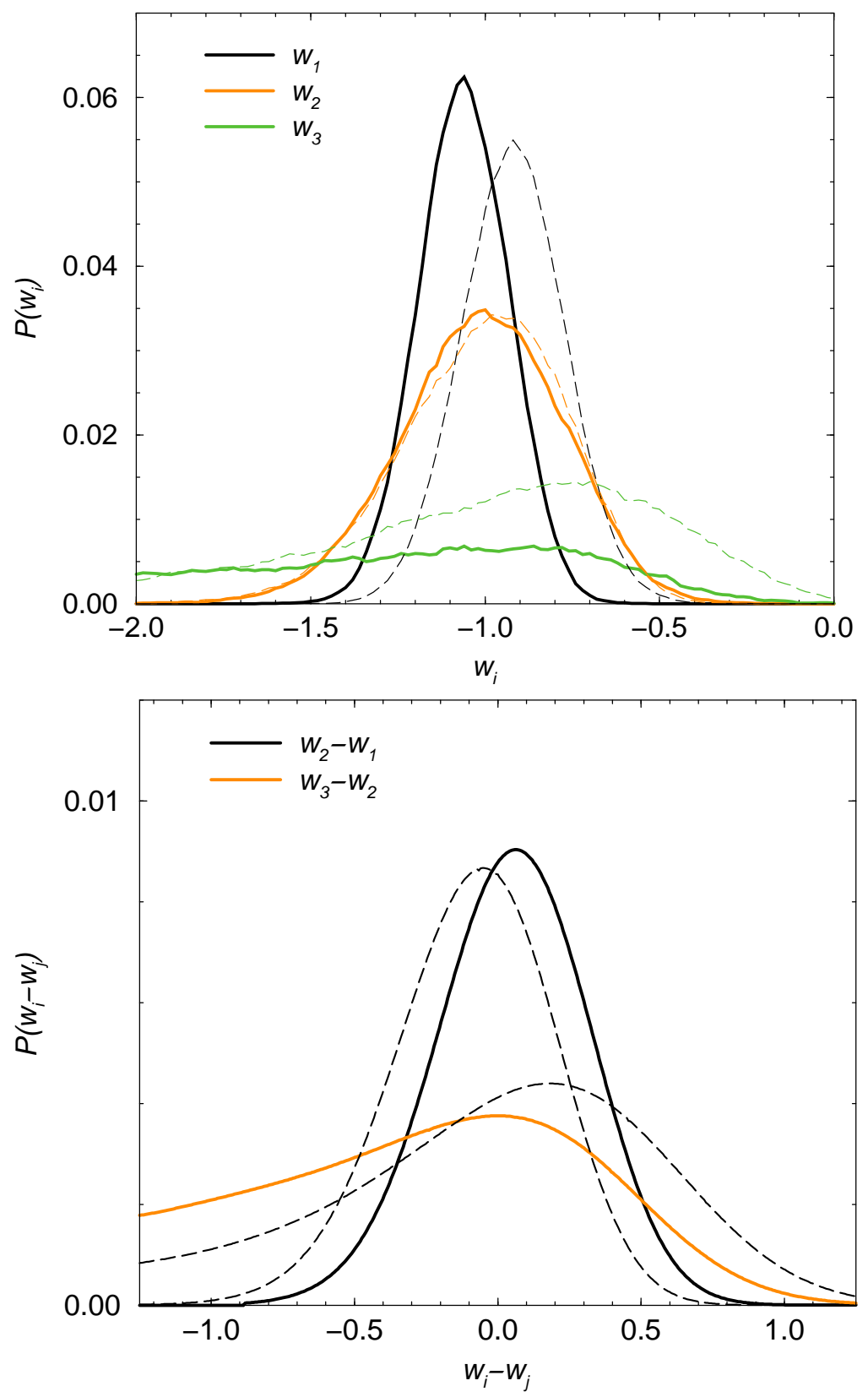

Figure 3. Probability distribution functions of uncorrelated estimates of the dark energy equation of state (top panel) and the difference between estimates of the equation of state (bottom panel). Solid and dashed lines are for the case with 192 and $104 \mathrm{SNe}$ (see caption of Figure 1), respectively.

are uncorrelated following the technique of Huterer \& Cooray [20] and as outlined in Section II. The procedure to decorrelate binned estimates that are predefined over a certain range usually results in adding contributions from nearby bins, but these are generally smaller than the main contribution from the bin in which the estimate was first defined. On average, the first uncorrelated measure is $73 \%$ determined by its own bin, with a minimal contribution from the third bin. The second measure, on average, was $50 \%$ determined by the second bin, with a substantial contribution from the first 


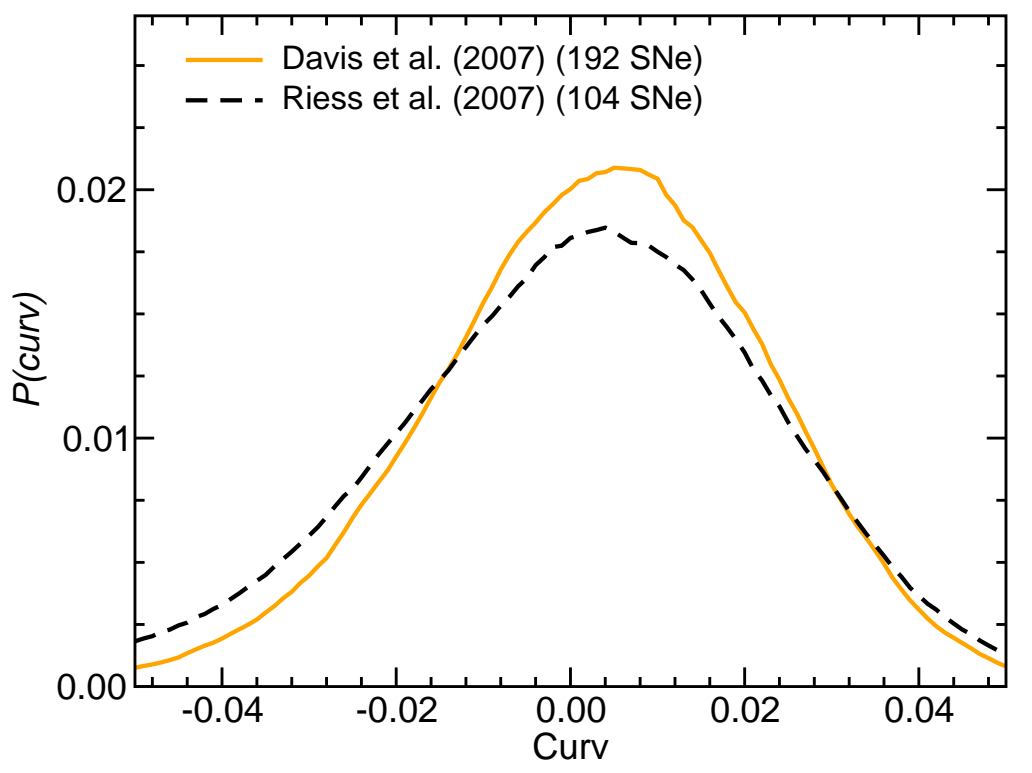

Figure 4. Probability distribution function of cosmic curvature by combining supernova data with additional constraints outlined in the paper. We assume a broad, flat prior for $\Omega_{k}$, but the combination of SN data and CMB and BAO distance scales results in a tight constraint on curvature. We find $\Omega_{k}=0.004 \pm 0.019(192 \mathrm{SNe})$ and $\Omega_{k}=0.002 \pm 0.022(104 \mathrm{SNe}$ ), which is fully consistent with estimates made by the WMAP analysis by combining WMAP data with supernova data from the SNLS survey.

bin. The third measure was typically $54 \%$ determined by the third bin, with a $6 \%$ contribution from the first bin.

These results do not presume a particular evolutionary history, as opposed to model fitting to a specific form, e.g. $w(z)=w_{0}+w_{1} z$ [16] or $w(z)=w_{0}+w_{a}(1-a)$. Our procedure, which fits binned values of $w\left(z_{i}\right)$ and then decorrelates them, has the advantage that one can extract redshift evolution independent of a model. This is particularly effective if the model to be assumed turns out not to be an accurate representation of the true underlying EOS.

As shown in Figure 2, the dark energy EOS as a function of redshift is fully consistent with $w(z)=-1$ at the $1 \sigma$ confidence level, for both the full sample of $192 \mathrm{SNe}$, and the subset of 104 SNe corresponding to the earlier analysis [3]. As shown in the lower panel of Figure 2, this conclusion is unchanged when we drop the assumption related to a flat cosmological model, regardless of the assumed prior on $\Omega_{k}$. Our external constraints related to distance to the last scattering surface from CMB, and BAO distance scale to $z=0.35$, provide a strong constraint on $\Omega_{k}$. We explicitly include an additional flatness constraint to allow comparison with earlier work [5, 3]. To highlight the extent to which EOS estimates are consistent with $w(z)=-1$, in Figure 3 we plot the probability distribution functions $P\left(w_{i}\right)$ both for flat cosmologies and for the case with curvature allowed to vary. Except for the third bin, which still remains mostly undefined with a very broad probability function, the first two bins are peaked and allow constraints 

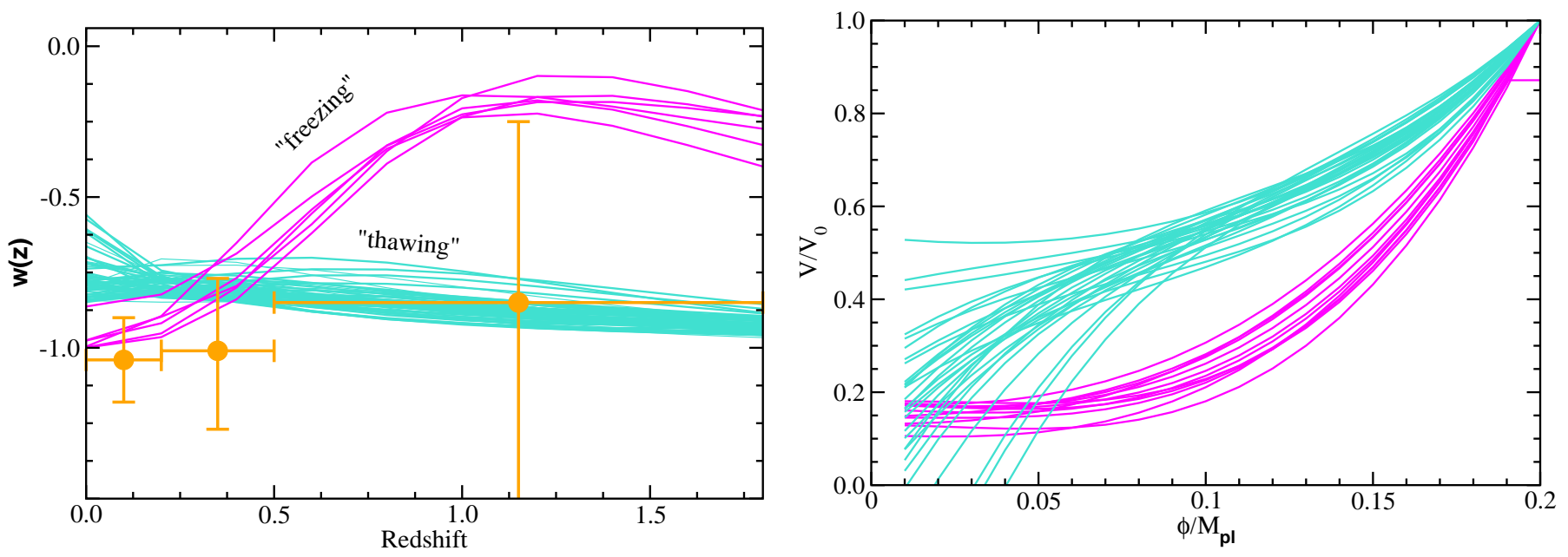

Figure 5. Left: A comparison of $w(z)$ estimates and dynamical dark energy models, based on the Monte-Carlo modeling approach of Ref. [28], that are inconsistent with current estimates. We show both cases of "thawing" and "freezing" models inconsistent with current data (see text for details). Right: The shapes of potentials generally corresponding to $w(z)$ models shown in the left panel which are inconsistent with our estimates of the binned EOS from a combined sample of supernovae and cosmological distance scale measurements. We show $V(\phi)$ as a function of the scalar field $\phi$ when potentials are normalized to the value at $z=3\left(V_{0} \equiv V(z=3)\right)$, which we take to be $\phi=0.2$.

at a high confidence level over $-2<w<0$. These show a clear consistency with $w=-1$, and hence are completely consistent with a cosmological constant. While the allowed range for $w\left(z_{3}\right)$ is broad, at the $68 \%$ confidence level we find that $w\left(z_{3}\right)<-0.2$, suggesting that we can rule out a large EOS even at $z>0.5$.

The uncorrelated binned estimates of $w(z)$ derived in Huterer \& Cooray [20] using an earlier "Gold" sample from Riess et al. [29] showed an equation of state that varied significantly between the lowest-redshift bin and the second bin. This difference decreased in the most recent "Gold" sample as analyzed by Riess et al. [3]. In the current work, utilizing an extended sample of supernovae, we no longer find evidence for a variation in the dark energy EOS between the first and the second bin. To show this explicitly, we also plot the probability distribution function of the difference between binned estimates of $w_{i}(z)$ in Figure 3. Between the first and the second bin we find the difference to be $w_{2}-w_{1}=0.06 \pm 0.26$ at the $68 \%$ confidence level for the 192 SNe sample with a flat model. Current data is thus completely consistent with a cosmological constant. Previous estimates of a large and a statistically significant value for $w_{2}-w_{1}$, with $w_{1}<-1$ and $w_{2}>-0.8$, led to suggestions in the literature for a physical mechanism called dark energy "metamorphosis" [30]. While earlier conclusions were limited to a small set of supernovae data, with the larger sample it is now clear that there is little evidence for a sudden transition in the EOS around $z \sim 0.2$. Future data could tighten these constraints, either further narrowing down to a cosmological constant, or providing evidence for small variations in the EOS with redshift. 

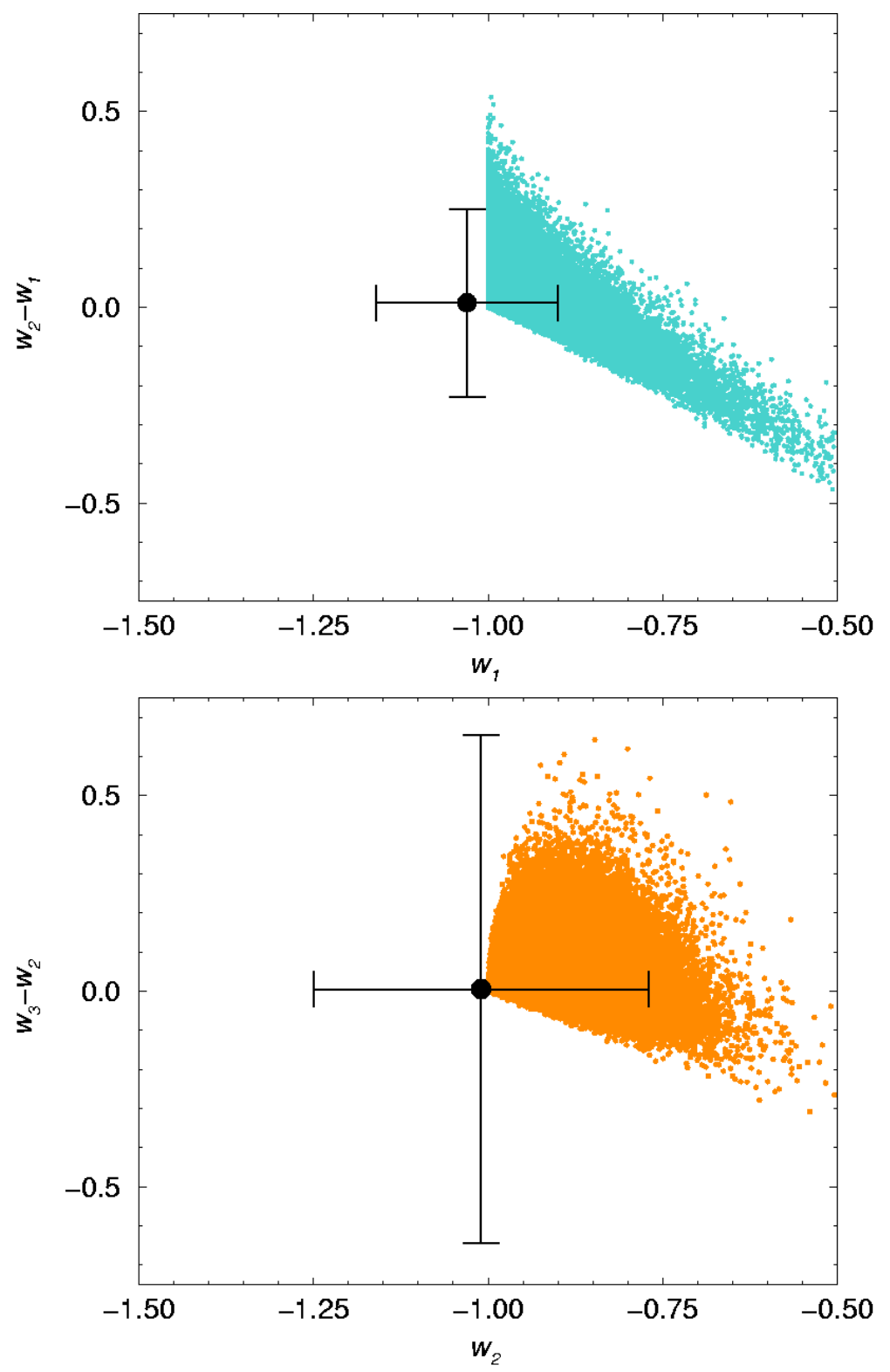

Figure 6. A comparison of $w(z)$ values from dynamical dark energy models, based on the Monte-Carlo modeling approach of Ref. 28. Each dot represents a potential dynamical dark energy model. The data points are the allowed range from our analysis.

We show that our conclusions are generally unchanged by assumptions related to the curvature. This is because we constrain the EOS using a combination of supernova data and existing measurements of the cosmic distance scale out to $z=1089$ and $z=0.35$ with $\mathrm{CMB}$ and $\mathrm{BAO}$, respectively. The combination of supernova data and these measurements, combined with our prior on the Hubble constant, leads to a strong independent constraint on the curvature parameter $\Omega_{k}$. We show the probability distribution $P\left(\Omega_{k}\right)$ in Figure 4 for both the full sample and the subset of 104 supernovae. In both cases $\Omega_{k}$ is consistent with zero; with the full supernovae sample, we find $\Omega_{k}=0.004 \pm 0.019$. This is about $1 \sigma$ away from the combined WMAP and Hubble 
constant estimate of $\Omega_{k}=-0.014 \pm 0.017$ or the combined WMAP+SNLS estimate of $\Omega_{k}=-0.011 \pm 0.012$ [7]. On the other hand, the combined WMAP+SDSS estimate from the same analysis is $\Omega_{K}=-0.0053_{-0.0060}^{+0.0068}$, which is a shift in the direction of the $\Omega_{k}$ value we find when the combined SNe dataset is analyzed with the WMAP and BAO priors.

To demonstrate how our estimates of $w\left(z_{i}\right)$ can be used to understand the redshift evolution of the dark energy component, in Figure 5 we show a sample of predictions related to dynamical dark energy models that are ruled out with present estimates of $w_{i}(z)$ at the $68 \%$ confidence level. These cases generally involve a dark energy EOS that starts as $w>-0.2$ at high redshifts, or an EOS that stars with a value around -1 at high redshift but evolve to a value greater than -0.85 when $z<0.2$. The former models belong to the general category of "freezing" models described in Caldwell \& Linder [21], while the latter models are categorized as "thawing" models. We generate these models following the numerical technique of Huterer \& Peiris [28], writing the KleinGordon equation for the evolution related to a scalar field as $\ddot{\phi}+3 H \dot{\phi}+d V / d \phi=0$, and then numerically generating a large number of models following the Monte-Carlo flow approach as used for numerical models of inflation [31, 32, 33, 34, 35]. We do not reproduce details as the process is similar to the modeling of Ref. [28, 36].

As a further application of our estimates of the EOS, and the difference between two binned estimates of EOS, in Figure 6 we compare our measured value with values expected for a large number of models. Again, we rule out certain extreme models where $w\left(z_{i}\right)$ varies rapidly between adjacent bins. In dynamical dark energy models, models that generally lead to a large variation in $w$ between two adjacent bins also have a value significantly different from - 1 in one of the bins. Thus most models are currently ruled out from the value of $w$ in a single bin, rather than through the difference of $w_{2}-w_{1}$ or $w_{3}-w_{2}$, since the the latter are still largely uncertain. While we can use the numerical modeling technique of Huterer \& Peiris [28] to make qualitative statements about the EOS, and to rule out extreme possibilities for its dynamical evolution, given the stochastic nature of model generation we cannot use this sort of method to make detailed statements about, for example, the scalar field (quintessence) potential responsible for dark energy. Instead, it is necessary to directly reconstruct the scalar field potential from supernovae distance data. While there are attempts to recover the potential by directly model fitting various parametric forms of the potential as a function of the scalar field, such as power-law or polynomial functions of the scalar field $\phi$, model independent binned estimates of the potential are preferable [37].

\subsection{A New Figure of Merit}

As discussed in the previous sections, our binned estimates allow us to study the redshift evolution of the dark energy EOS without the need to assume an underlying model. This is to be contrasted with the usual approach, in which a parameterized form for $w(z)$ is required to fit the data. With an increasing supernova sample size, and improvement 
in other cosmological observations, we may be able to recover three or more binned values at the $10 \%$ level or better. In the context of planning dark energy experiments, and assessing the constraining power of future data, it may be advantageous to consider binned estimates of the dark energy EOS, rather than the error associated with a specific and arbitrary two parameter model of the equation of state. The latter is the approach adopted to quote the "figure of merit" (FOM) of an experiment, based on the inverse of the area of the ellipse of the two parameters describing the EOS with redshift (introduced in [23]). In the case of binned estimates, once uncorrelated, we can quote an alternative FOM as $\left[\sum_{i} 1 / \sigma^{2}\left(w_{z_{i}}\right)\right]^{1 / 2}$, which takes into account the combined inverse variance of all independent estimates of the EOS. In an upcoming paper we will quantify the exact number of $w(z)$ estimates that can be determined with future experiments involving supernovae and large-scale structure (weak lensing, baryon acoustic oscillations over a wide range in redshift), and we will compare this alternative FOM to the method of Ref. [23].

\section{Summary}

We use a sample of 192 SNe Ia (and a subset for comparison) to constrain the dark energy equation-of-state parameter and its variation as a function of redshift. We use a model independent approach, providing uncorrelated measurements across three redshift bins below $z=1.8$, and find that $w(z)$ is consistent with a cosmological constant $(w(z)=-1)$ at the $68 \%$ confidence level. At the same confidence level we find that the EOS is greater than -0.2 over the redshift range of $0<z<1.8$. Overall, there is no strong evidence against the assumption of a flat $\Omega_{k}=0$ universe, especially when recent supernova data are combined with cosmological distance scale measurements from WMAP and BAO experiments. We argue against previous claims in the literature of evolving dark energy, such as dark energy "metamorphosis", where the EOS changes significantly from $w>-1$ at $z>0.2$ to $w<-1$ at $z<0.2$. Instead, we find consistency with a cosmological constant, encapsulated in the $68 \%$ level constraint: $w_{2}-w_{1}=0.06 \pm 0.26$, where $w_{1}$ is the value of the dark energy EOS in the $z<0.2 \mathrm{bin}$, and $w_{2}$ is the value in the bin $0.2<z<0.5$. A transition in the EOS can also be ruled out between our second and third binned estimates of EOS, although we still find large uncertainties in our determination of the EOS at $z>0.5$, and we are insensitive to rapid variations at $z>1$. We compare our EOS estimates to Monte Carlo generated dynamical dark energy models associated with a single scalar field potential. Our EOS estimates generally allow us to rule out extreme "thawing" and "freezing" models, though a large number of potential shapes remain in agreement with current data.

We also suggest an alternative, parameter independent figure-of-merit, with which to evaluate the potential of future missions to constrain properties of the dark energy. 


\section{Acknowledgments}

$\mathrm{AC}$ and DEH are partially supported by the DOE at LANL through IGPP grant Astro-1603-07. AC acknowledges funding for undergraduate research, at UC Irvine as part of NSF Career AST-0645427, which was used to support SS, and thanks UCI Undergraduate Research Opportunities Program (UROP) for additional support in the form of a UCI Chancellor's Award for Fostering of Distinguished Undergraduate Research. DEH acknowledges a Richard P. Feynman Fellowship from LANL.

\section{References}

[1] A. G. Riess et al., B. J. Barris et al., arXiv:astro-ph/0310843 R. A. Knop et al., arXiv:astro-ph/0309368, J. L. Tonry et al., Astrophys. J. 594, 1 (2003).

[2] S. Perlmutter et al., Astrophys. J. 517, 565 (1999); A. Riess et al., Astron. J. 116, 1009 (1998).

[3] A. G. Riess et al., arXiv:astro-ph/0611572.

[4] W. M. Wood-Vasey et al., arXiv:astro-ph/0701041.

[5] T. M. Davis et al., arXiv:astro-ph/0701510.

[6] D. N. Spergel et al. [WMAP Collaboration], Astrophys. J. Suppl. 148, 175 (2003);

[7] D. N. Spergel et al. [WMAP Collaboration], arXiv:astro-ph/0603449

[8] T. Padmanabhan, Phys. Rept. 380, 235 (2003) arXiv:hep-th/0212290.

[9] P. Astier et al., Astron. Astrophys. 44731 (2006), astro-ph/0510447.

[10] R. R. Caldwell, R. Dave and P. J. Steinhardt, Phys. Rev. Lett. 80, 1582 (1998) arXiv:astro-ph/9708069.

[11] P. M. Garnavich et al. [Supernova Search Team Collaboration], Astrophys. J. 509, 74 (1998) arXiv:astro-ph/9806396.

[12] M. Visser, Class. Quant. Grav. 21, 2603 (2004) arXiv:gr-qc/0309109.

[13] D. Huterer and M. S. Turner, Phys. Rev. D60 081301 (1999).

[14] M. Sahlen, A. R. Liddle and D. Parkinson, Phys. Rev. D 72, 083511 (2005) arXiv:astro-ph/0506696.

[15] C. Li, D. E. Holz and A. Cooray, Phys. Rev. D 75, 103503 (2007) arXiv:astro-ph/0611093.

[16] A. R. Cooray and D. Huterer, Astrophys. J. 513, L95 (1999) arXiv:astro-ph/9901097.

[17] E.V. Linder, Phys. Rev. Lett. 90091301 (2003).

[18] B. F. Gerke and G. Efstathiou, "Probing quintessence: Reconstruction and parameter estimation from Mon. Not. Roy. Astron. Soc. 335, 33 (2002) arXiv:astro-ph/0201336.

[19] D. Huterer and G. Starkman, Phys. Rev. Lett. 90, 031301 (2003).

[20] D. Huterer and A. Cooray, Phys. Rev. D 71, 023506 (2005) arXiv:astro-ph/0404062.

[21] R. R. Caldwell and E. V. Linder, Phys. Rev. Lett. 95, 141301 (2005) arXiv:astro-ph/0505494].

[22] Y. Wang and M. Tegmark, Phys. Rev. D 71, 103513 (2005) arXiv:astro-ph/0501351.

[23] A. Albrecht et al., arXiv:astro-ph/0609591.

[24] M. Tegmark et al. [SDSS Collaboration], Phys. Rev. D 69, 103501 (2004) arXiv:astro-ph/0310723].

[25] A. G. Riess et al., Astrophys. J. 627, 579 (2005) arXiv:astro-ph/0503159].

[26] D. J. Eisenstein et al. [SDSS Collaboration], Astrophys. J. 633, 560 (2005) arXiv:astro-ph/0501171.

[27] Y. Wang and P. Mukherjee, arXiv:astro-ph/0703780.

[28] D. Huterer and H. V. Peiris, arXiv:astro-ph/0610427.

[29] A. G. Riess et al. [Supernova Search Team Collaboration], Astrophys. J. 607, 665 (2004) arXiv:astro-ph/0402512.

[30] U. Alam, V. Sahni, T. D. Saini and A. A. Starobinsky, Mon. Not. Roy. Astron. Soc. 354, 275 (2004) arXiv:astro-ph/0311364.

[31] W. H. Kinney, Phys. Rev. D 66, 083508 (2002) arXiv:astro-ph/0206032]. 
[32] A. R. Liddle, Phys. Rev. D 68, 103504 (2003) arXiv:astro-ph/0307286.

[33] H. Peiris and R. Easther, JCAP 0607, 002 (2006) arXiv:astro-ph/0603587.

[34] S. Chongchitnan and G. Efstathiou, Phys. Rev. D 72, 083520 (2005) arXiv:astro-ph/0508355.

[35] B. C. Friedman, A. Cooray and A. Melchiorri, Phys. Rev. D 74, 123509 (2006) arXiv:astro-ph/0610220.

[36] S. Chongchitnan and G. Efstathiou, arXiv:0705.1955 [astro-ph].

[37] C. Li, D. E. Holz and A. Cooray, Phys. Rev. D 75, 103503 (2007) arXiv:astro-ph/0611093.

[38] M. Tegmark et al. [SDSS Collaboration], Astrophys. J. 606, 702 (2004) arXiv:astro-ph/0310725. 